\title{
Formation of Difluorobenzyl Radicals from 2,3,4-Trifluorotoluene in Corona Excitation
}

\author{
Young Wook Yoon, Gi Woo Lee, ${ }^{\text {a }}$ and Sang Kuk Lee* \\ Department of Chemistry and The Chemistry Institute for Functional Materials, Pusan National University, \\ Pusan 609-735, Korea. *E-mail: sklee@pusan.ac.kr \\ Received April 6, 2011, Accepted May 6, 2011
}

\begin{abstract}
The vibronically well-resolved emission spectrum was recorded from the corona discharge of precursor 2,3,4trifluorotoluene in a corona excited supersonic expansion with a pinhole-type glass nozzle using a long-path monochromator in the visible region. From the analysis of the observed spectrum, we found the evidence of the presence of the difluorobenzyl radicals in the corona discharge of the precursor. A possible mechanism is proposed for the formation of difluorobenzyl radicals in the gas phase on the basis of the observed emission intensity of the difluorobenzyl radicals produced.
\end{abstract}

Key Words : Spectroscopy, Difluorobenzyl radical, Reaction mechanism

\section{Introduction}

Transient species such as molecular ions and radicals have long been believed to play an important role as intermediates in chemical reactions. ${ }^{1}$ For those interested in the reaction dynamics at the transition state, the characteristics and structure of the transient species may serve at least to describe the nature of the chemical reactivity. ${ }^{2}$

The benzyl radical, the prototype aromatic radical, is one of the fundamental reaction intermediates in aromatic chain reactions and has been the subject of numerous spectroscopic works for a long time. ${ }^{3}$ With the background of the delocalized $\pi$ electronic structure of the benzyl radical, many benzyl-type radicals have been extensively studied to explain their substitution effect by employing a variety of experimental schemes, in which the technique of corona excited supersonic expansion (CESE) has been proved to be a useful tool for the observation of the jet-cooled benzyltype radicals. ${ }^{4-10}$ In this group, we significantly improved the generation efficiency of benzyl-type radicals by upgrading the nozzle system ${ }^{11}$ for the spectroscopic observation of transient species.

The spectroscopic investigation of fluorobenzyl radicals was initiated by Bindley et al. using an electric discharge of the corresponding fluorotoluenes, ${ }^{12}$ in which all three isomers of fluorobenzyl radicals were clearly identified from the analysis of the vibronic emission spectra. The laser induced fluorescence (LIF) spectra of fluorobenzyl radicals were recorded by monitoring the emission from the decomposition products of the suitable precursors. ${ }^{13,14}$ For high resolution spectroscopic work, the gas phase spectrum of the $p$-fluorobenzyl radical observed at room temperature ${ }^{15}$ was simulated to determine the change of their rotational constants upon electronic excitation. Cossart-Magos and Cossart

a Present address: Department of Chemistry, Korea University, Seoul, Korea obtained accurate rotational constants from the analysis of the laser excitation spectra of the jet-cooled $p$-fluorobenzyl radicals. ${ }^{16}$

In this laboratory, we observed for a long time the vibronic emission spectra of many fluorine substituted benzyl-type radicals, including mono-, ${ }^{17-19}$ bi-,${ }^{6,20-24}$ and penta- ${ }^{25}$ fluorine substituted products. From the analysis of the observed vibronic spectra of difluorobenzyl radicals, ${ }^{6}$ we identified spectroscopic evidence of the internal molecular rearrangement of fluorine and the methylene group, making it possible to convert into more stable isomers. Similar phenomena was observed in the corona discharge of 2,6-dichlorotoluene ${ }^{26}$ using a pinhole-type nozzle.

In this paper, we present the observation of the visible vibronic emission spectrum from the corona discharge of the precursor 2,3,4-trifluorotoluene in CESE, in which the existence of the jet-cooled difluorobenzyl radicals was spectroscopically identified. From the analysis of the emission intensity observed, a possible mechanism is proposed for the formation of difluorobenzyl radicals from the precursor.

\section{Experimental}

The experimental setup employed in this work is similar to those described elsewhere. ${ }^{27} \mathrm{~A}$ corona excited supersonic expansion (CESE) technique was employed using a pinholetype glass nozzle for the excitation and formation of benzyltype radicals in corona discharge, and a long path monochromator for the observation of the vibronic emission spectra.

The benzyl-type radicals were generated at the excited vibronic states by the corona discharge of precursor 2,3,4trifluorotoluene (Aldrich, reagent grade) with a large amount of the inert carrier gas He. The vapor of the precursor at room temperature was mixed with 2.0 bar of $\mathrm{He}$ gas inside the vaporizing vessel. The concentration of the precursor vapor in the gas mixture was adjusted by controlling the 
openness of the bypass valve to achieve the maximum emission intensity monitored from the strongest band in the spectrum. Since the heavy aromatic compounds produce a soot deposit clogging the orifice of the glass nozzle during the corona discharge, we adopted a modified pinhole-type nozzle with a diameter of $0.3 \mathrm{~mm}$ made in this laboratory, which significantly improved the long period stability of the corona discharge. A discharge voltage of approximately 1.5 $\mathrm{kV}$ and a current of $3 \mathrm{~mA}$ were applied to achieve the maximum emission intensity from the benzyl-type radicals, as well as the least production of small fragments, such as $\mathrm{C}_{2}$ and $\mathrm{CH}$ radicals which also emit strong fluorescence in the visible region.

The Pyrex 6-way expansion chamber was evacuated by a mechanical vacuum pump, maintaining a chamber pressure of about 1.0 mbar during the continuous expansion with a backing pressure of 3.0 bar. Upon the electric DC discharge of the precursor, a blue-green colored jet appeared, indicating the formation of benzyl-type radicals. The emission from the jet area of $5 \mathrm{~mm}$ below the nozzle throat was collected through the quartz lens $(\mathrm{f}=50 \mathrm{~mm}, \mathrm{~d}=38 \mathrm{~mm}$ ) placed inside the chamber arm and focused onto the slit of the monochromator (Jobin Yvon U1000) with a cooled PMT (Hamamatsu R649) detector. The vibronic emission spectrum was recorded by scanning from 18000 to $22500 \mathrm{~cm}^{-1}$ with steps of $2.0 \mathrm{~cm}^{-1}$ with a slit width of $100 \mu \mathrm{m}$ for $1 \mathrm{hr}$. The wavenumber of the spectrum was calibrated by the He atomic lines ${ }^{28}$ recorded at the same time as the spectrum and believed to be accurate to within $\pm 1.0 \mathrm{~cm}^{-1}$.

\section{Results and Discussion}

It has been demonstrated that a well-controlled corona discharge of substituted toluene with a large amount of inert carrier gas produces the corresponding benzyl-type radicals in the vibronically excited state. Although the exact mechanism for the generation and excitation of benzyl-type radicals has not been well established in a CESE using a pinhole-type nozzle, it has been suggested that the metastable He atom in the $1 \mathrm{~s} 2 \mathrm{~s}{ }^{3} \mathrm{~S}_{1}$ state, above approximately $19.82 \mathrm{eV}$, initially excited by the corona discharge, transfers its excess energy to the precursor through collisional process. Subsequently, one of the $\mathrm{C}-\mathrm{H}$ bonds of the methyl group, already loosely bonded in the excited electronic state, is dissociated to generate the corresponding benzyl-type radicals by eliminating a hydrogen atom.

The molecular radical that is generated undergoes collisional relaxation with the $\mathrm{He}$ atoms in the process of losing its rotational and vibrational energy in the excited electronic states, producing electronically hot but rovibrationally cold species. A simulation of the rotational contour showed a rotational temperature ${ }^{4}$ of about $40 \mathrm{~K}$, which is relatively high for supersonic jet expansion because of the electric dc discharge, continuous jet expansion, and low backing pressure, in which the maximum pressure is limited by the tolerance of the glassware used for the nozzle.

In benzyl-type radicals that have a planar structure with 7 delocalized $\pi$ electrons, the electronic interaction between the substituents and the benzene ring should be undoubtedly of the second-order compared with that between the methylene group and the benzene ring, to which the methylene group contributes a delocalized $\pi$ electron. Thus, the electronic structure of the benzyl-type radicals should be similar to that of the parental benzyl radical, and one might be able to closely relate the two lowest-lying electronic states of benzyl-type radicals to the $2^{2} \mathrm{~B}_{2}\left(\mathrm{D}_{2}\right)$ and $1^{2} \mathrm{~A}_{2}\left(\mathrm{D}_{1}\right)$ states of the benzyl radical. ${ }^{29}$

The visible emission of benzyl-type radicals is believed to arise from transitions to the $1^{2} \mathrm{~B}_{2}\left(\mathrm{D}_{0}\right)$ ground state from the close-lying $\mathrm{D}_{2}$ and $\mathrm{D}_{1}$ excited electronic states which could be mixed through vibronic coupling. ${ }^{29}$ Ring substitution is also expected to affect the energies of the two excited electronic states differently. However, it is extremely difficult to observe the transition from the $\mathrm{D}_{2}$ to $\mathrm{D}_{0}$ states, because of the efficient collisional relaxation from the $D_{2}$ to $D_{1}$ states through the vibronic coupling. Thus, the vibronic emission spectrum observed with CESE using a pinhole-type glass nozzle is similar to the dispersed fluorescence spectra obtained while exciting the origin band of the $\mathrm{D}_{1} \leftarrow \mathrm{D}_{0}$ electronic transition, providing the origin band with the strongest intensity at the highest wavenumber. The transition from the ground vibrational state of the excited electronic state to the ground electronic state provides information on the electronic transition energy as well as the vibrational mode frequencies in the ground electronic state, which is very useful for identification of the species generated in the corona discharge of precursor.

The fluorine-substituted benzyl radicals exhibit red-shifted origin bands compared to the benzyl radical at $22002 \mathrm{~cm}^{-1}$. The origin bands of the $o-, m$-, and $p$-fluorobenzyl radicals ${ }^{5}$ are observed at 21924,21691 , and $21527 \mathrm{~cm}^{-1}$, which are shifted by 78,311 , and $475 \mathrm{~cm}^{-1}$ from those of the benzyl radical, ${ }^{3}$ respectively. Also, the origin bands of the difluoro-

Table 1. Origin bands of fluorine substituted benzyl radicals in the $\mathrm{D}_{1} \rightarrow \mathrm{D}_{0}$ transition $^{a}$

\begin{tabular}{|c|c|c|}
\hline Molecules & Origin band & Shift $^{d}$ \\
\hline benzyl $^{b}$ & 22002 & 0 \\
\hline$o$-fluorobenzyl ${ }^{c}$ & 21924 & 78 \\
\hline$m$-fluorobenzyl ${ }^{c}$ & 21691 & 311 \\
\hline$p$-fluorobenzyl $l^{c}$ & 21527 & 475 \\
\hline 2,3-difluorobenzyl ${ }^{e}$ & 21338 & 664 \\
\hline 2,4-difluorobenzyl ${ }^{f}$ & 21846 & 156 \\
\hline 2,5-difluorobenzyl ${ }^{g}$ & 21048 & 954 \\
\hline 2,6-difluorobenzyl ${ }^{h}$ & 21774 & 228 \\
\hline 3,4-difluorobenzyl $l^{i}$ & 21962 & 40 \\
\hline 3,5-difluorobenzyl ${ }^{j}$ & 21182 & 820 \\
\hline$p$-fluoro- $\alpha$-methylbenzyl ${ }^{k}$ & 20854 & 1148 \\
\hline pentafluorobenzyl $^{l}$ & 21857 & 145 \\
\hline
\end{tabular}

${ }^{a}$ Measured in vacuum $\left(\mathrm{cm}^{-1}\right) \cdot{ }^{b}$ Reference $3 .{ }^{c}$ Reference $5 .{ }^{d}$ With respect to the origin band of benzyl radical $\left(22002 \mathrm{~cm}^{-1}\right)$. ${ }^{e}$ Reference 23 . ${ }^{f}$ Reference 21. ${ }^{g}$ Reference $24 .{ }^{h}$ Reference $20 .{ }^{i}$ Reference $22 .{ }^{j}$ Reference 6. ${ }^{k}$ Reference $7 .{ }^{~}$ Reference 25 . 


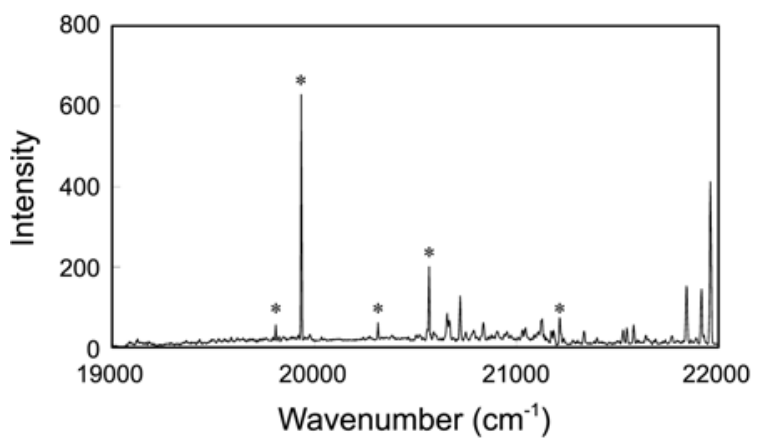

Figure 1. A portion of the vibronic emission spectrum observed from the corona discharge of 2,3,4-trifluorotoluene seeded in a large amount of an inert carrier gas helium, in a corona excited supersonic expansion using a pinhole-type nozzle. Atomic lines are marked by an asterisk in the spectrum.

benzyl radicals were observed to be red shifted compared to the benzyl radical. It has recently been reported that the $p$ fluoro- $\alpha$-methylbenzyl radical ${ }^{7}$ exhibits a large shift of 1148 $\mathrm{cm}^{-1}$ compared to the benzyl radical, which suggests that there might be a synergic effect for the extension of the $\pi$ electrons delocalized over additional substituents. The positions of the origin bands of fluorine substituted benzyltype radicals in the $\mathrm{D}_{1} \rightarrow \mathrm{D}_{0}$ electronic transition are listed in Table 1.

Figure 1 shows a portion of the visible vibronic emission spectrum observed from the corona discharge of precursor

Table 2. List of the vibronic bands observed and their assignments ${ }^{a}$

\begin{tabular}{|c|c|c|}
\hline Position & Intensity & Assignments $^{b}$ \\
\hline 21960 & vs & Origin of 3,4 \\
\hline 21916 & $\mathrm{~s}$ & \\
\hline 21844 & s & Origin of 2,4- \\
\hline 21772 & W & \\
\hline 21582 & $\mathrm{~m}$ & \\
\hline 21546 & $\mathrm{~m}$ & \\
\hline 21530 & $\mathrm{~m}$ & $16 b_{1}^{0}$ of $3,4-$ \\
\hline 21338 & $\mathrm{w}$ & Origin of 2,3- \\
\hline 21216 & $\mathrm{~m}$ & He atomic \\
\hline 21186 & w & $12_{1}^{0}$ of $3,4-$ \\
\hline 21128 & $\mathrm{~m}$ & $1_{1}^{0}$ of $2,4-$ \\
\hline 21048 & $\mathrm{w}$ & $12_{1}^{0}$ of $2,4-$ \\
\hline 20912 & $\mathrm{w}$ & $7 b_{1}^{0}$ of 2,4 \\
\hline 20838 & $\mathrm{w}$ & $6 a_{1}^{0}$ of $2,3-$ \\
\hline 20794 & $\mathrm{w}$ & $6 b_{1}^{0}$ of $2,3-$ \\
\hline 20754 & $\mathrm{w}$ & \\
\hline 20722 & $\mathrm{~m}$ & \\
\hline 20672 & $\mathrm{w}$ & $7 a_{1}^{0}$ of $3,4-$ \\
\hline 20658 & $\mathrm{~m}$ & $1_{1}^{0}$ of 2,3 \\
\hline 20570 & $\mathrm{~s}$ & $\mathrm{H}$ atomic \\
\hline 20316 & $\mathrm{~m}$ & He atomic \\
\hline 19938 & vs & He atomic \\
\hline 19810 & W & He atomic \\
\hline
\end{tabular}

${ }^{a}$ Measured in vacuum $\left(\mathrm{cm}^{-1}\right) .{ }^{b}$ The notation of vibrational modes in difluorobenzyl radicals as well as atomic lines. 2,3,4-trifluorotoluene seeded in a large amount of an inert carrier gas He in CESE. Most of the bands were observed with a very good $\mathrm{S} / \mathrm{N}$ in the spectral region of 19000-22000 $\mathrm{cm}^{-1}$, as listed in Table 2. In contrast with the typical spectra observed from the benzyl-type radicals using a pinhole-type glass nozzle, however, several strong bands appeared in the vicinity of the strongest band near $22000 \mathrm{~cm}^{-1}$, indicating that more than one benzyl-type radical species exist in the corona discharge of precursor. The several bands not assigned in Table 2 may belong to the 2,3,4-trifluorobenzyl radical generated by loss of a hydrogen atom from the methyl group of the precursor. However, the assignment of the bands cannot proceed due to the lack of vibrational information of the precursor.

For the identification of the species generated in the corona discharge, we compared this spectrum with that of the difluorobenzyl radicals in Figure 2, thanks to the previous works done in this laboratory. The strongest origin bands of the 3,4-, 2,4-, and 2,3-difluorobenzyl radicals were coincided with the observed bands, confirming that a few difluorobenzyl radicals were produced in the corona discharge of the precursor. While the dissociation of one of the $\mathrm{C}-\mathrm{H}$ bonds of the methyl group in the excited electronic state produces the trifluorobenzyl radical and a free hydrogen atom, as discussed in the generation of the $o$-xylyl radical ${ }^{30}$ from $o$-xylene, the difluorobenzyl radicals are believed to be produced from the intramolecular replacement of a fluorine atom of the precursor by a hydrogen atom of the methyl group at the excited electronic state. Since there is no solvent effect in the gas phase reaction, the intermolecular replacement of a fluorine atom in the 2,3,4-trifluorobenzyl radical by a free hydrogen atom is not a feasible pathway in this reaction because the steric factor of the collision theory seems to be very small in a large aromatic compound. ${ }^{31}$

Instead, we suggest that the formation of difluorobenzyl radicals is the result of the direct replacement of a fluorine atom through its intramolecular reaction with a hydrogen atom of the methyl group. The very strong, strong, and very weak observation of the origin bands of the 3,4,- 2,4-, and

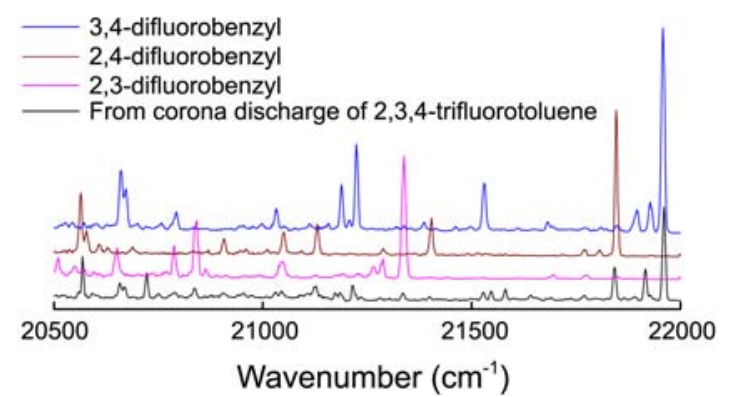

Figure 2. Comparison of the spectra observed from difluorobenzyl radicals with that from the corona discharge of 2,3,4-trifluorotoluene. Several strong bands of the 2,3-, 2,4-, and 3,4-difluorobenzyl radicals are coincident with those from the corona discharge of 2,3,4-trifluorotoluene, confirming the formation of difluorobenzyl radicals from the precursor. The origin bands of 3,4-, 2,4, and 2,3isomers show very strong, strong, and weak intensity in the observed spectrum, respectively. 


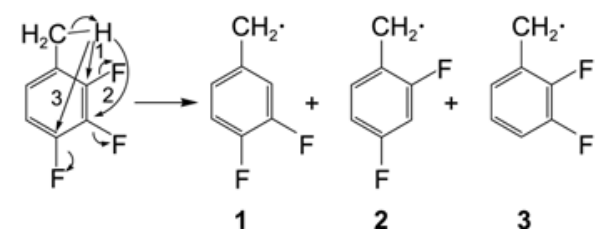

Figure 3. Proposed mechanism for formation of difluorobenzyl radicals from precursor. The difluorobenzyl radicals are formed by the direct displacement of a fluorine atom by a hydrogen atom of the methyl group. The displacement of a fluorine atom by a hydrogen atom at the 2,3 , and 4-positions produces [1]3,4-, [2]2,4-, and [3]2,3-difluorobenzyl radicals, respectively.

2,3-difluorobenzyl radicals in the spectrum, respectively, strongly suggests that the distance between the hydrogen atom of the methyl group and the carbon atom bonded to the fluorine atom plays a crucial role in the intramolecular substitution reaction, as shown in Figure 3.

Recently, the evidence of isomerization ${ }^{32}$ of the 3,5-difluorobenzyl radical was observed in the corona discharge of the 3,5-difluorotoluene with a large amount of inert carrier gas He. The 3,5-difluorobenzyl radical, the least stable species among the difluorobenzyl radicals, undergoes the internal rearrangement of the fluorine atom or methylene group to produce more stable 2,5-, 3,4-, and 2,4-difluorobenzyl radicals at the transition state. In this process, it was suggested that the stability of the intermediate species, a cyclic triangular structure, plays an important role in the production of isomers. The 2,4-difluorobenzyl radical formed from the migration of the methylene group exhibits the strong intensity in the spectrum, while the 3,4- and 2,5isomers produced by the movement of the fluorine atom result in medium intensity because the cyclic triangular intermediate using the methylene group has higher stability. However, the evidence of backward reaction, formation of the 3,5-difluorobenzyl radical in the corona discharge of 2,4-, 3,4-, and 2,5-difluorotoluenes, was not observed confirming that the key factor determining the pathway in the transition state is the stability of the intermediate species.

Very recently, Lee group reported the strong formation ${ }^{26}$ of the o-chlorobenzyl radical, as well as the 2,6-dichlorobenzyl radical, from the corona discharge of 2,6-dichlorotoluene, in which the easy elimination of the chlorine atom at the $o$-position is believed to be the result of the direct intramolecular replacement of the chlorine atom by a hydrogen atom of the methyl group, which is very similar to the mechanism described in this discussion.

In summary, we observed the vibronic emission spectrum from the corona discharge of precursor 2,3,4-trifluorotoluene, with a large amount of inert carrier gas He, in corona excited supersonic expansion using a pinhole-type glass nozzle. From the observed spectrum, we identified the formation of the 3,4-, 2,4-, and 2,3-difluorobenzyl radicals with a quite different intensity. A possible mechanism is proposed to describe the formation of difluorobenzyl radicals from the precursor, in which a hydrogen atom of the methyl group directly displaces the fluorine atom in the gas phase. It is believed that distance between the hydrogen atom and fluorine is the key factor determining the reaction rate.

Acknowledgments. This work was supported for two years by a Pusan National University Research Grant.

\section{References}

1. Carrington, A. Microwave Spectroscopy of Free Radicals; Academic Press: London, U.K., 1974.

2. Tan, X. Q.; Wright, T. G.; Miller, T. A. Electronic Spectroscopy of Free Radicals in Supersonic Jets: Jet Spectroscopy and Molecular Dynamics; Hollas, J. M.; Phillip, D., Eds.; Blackie Academic \& Professional: London, 1994.

3. Selco, J. I.; Carrick, P. G. J. Mol. Spectrosc. 1989, 137, 13.

4. Suh, M. H.; Lee, S. K.; Rehfuss, R. D.; Miller, T. A.; Bondybey, V. E. J. Phys. Chem. 1991, 95, 2727.

5. Selco, J. I.; Carrick, P. G. J. Mol. Spectrosc. 1995, 173, 277.

6. Lee, S. W.; Yoon, Y. W.; Lee, S. K. J. Phys. Chem. A 2010, 114, 9110.

7. Lee, G. W.; Lee, S. K. Chem. Phys. Lett. 2009, 470, 54.

8. Ahn, H. G.; Lee, G. W.; Lee, S. K. J. Phys. Chem. A 2008, 112, 13427.

9. Lee, G. W.; Lee, S. K. J. Chem. Phys. 2007, 126, 214.

10. Lee, G. W.; Lee, S. K. J. Phys. Chem. A 2007, 111, 6003.

11. Lee, S. K. Chem. Phys. Lett. 2002, 358, 110.

12. Bindley, T. F.; Watts, A. T.; Walker, S. Trans. Faraday Soc. 1964, $60,1$.

13. Charlton, T. R.; Thrush, B. A. Chem. Phys. Lett. 1986, $125,547$.

14. Fukushima, M.; Obi, K. J. Chem. Phys. 1990, 93, 8488.

15. Cossart-Magos, C.; Cossart, D.; Leach, S. Chem. Phys. 1973, 1, 306.

16. Cossart-Magos, C.; Cossart, D. Mol. Phys. 1988, 65, 627.

17. Lee, S. K.; Baek, D. Y. Chem. Phys. Lett. 1999, 301, 407.

18. Lee, S. K.; Ahn, B. U. Chem. Phys. Lett. 2000, 321, 25.

19. Lee, S. K.; Lee, S. K. J. Phys. Chem. A 2001, 105, 3034.

20. Lee, S. K.; Baek, D. Y. J. Phys. Chem. A 2000, 104, 5219.

21. Lee, G. W.; Lee, S. K. Chem. Phys. Lett. 2006, 430, 8.

22. Lee, G. W.; Lee, S. K. Chem. Phys. Lett. 2007, 440, 36.

23. Lee, G. W.; Ahn, H. G.; Kim, T. K.; Lee, S. K. Chem. Phys. Lett. 2007, 447, 197.

24. Ahn, H. G.; Lee, G. W.; Kim, T. K.; Lee, S. K. Chem. Phys. Lett. 2008, 454, 207.

25. Lee, S. K.; Baek, D. Y. Chem. Phys. Lett. 1999, 311, 36.

26. Yoon, Y. W.; Lee, S. W.; Lee, S. K. Bull. Korean Chem. Soc. 2010, $31,2479$.

27. Han, M. S.; Choi, I. S.; Lee, S. K. Bull. Korean Chem. Soc. 1996, $17,991$.

28. Weise, M. L.; Smith, M. W.; Glennon, B. M. Atomic Transition Probabilities; NSRD-NBS4, 1966.

29. Hiratsuka, H.; Mori, K.; Shizuka, H.; Fukushima, M.; Oki, K. Chem. Phys. Lett. 1989, 157, 35.

30. Fujiwara, M.; Tanimoto, Y. J. Phys. Chem. 1994, 98, 5695.

31. Atkins, P. W. Physical Chemistry, $6^{\text {th }}$ ed.; Oxford University Press: Oxford, 1998.

32. Yoon, Y. W.; Lee, S. W.; Lee, S. K. Bull. Korean Chem. Soc. 2010, $31,2783$. 